\title{
Integrative analysis of transcriptomics and metabolomics profiling on flesh quality of large yellow croaker Larimichthys crocea fed a diet with hydroxyproline supplementation
}

\author{
Zehong Wei ${ }^{1}$, Huihui Zhou ${ }^{1}$, Yanjiao Zhang ${ }^{1}$, Qin Zhang ${ }^{2}$, Wenbing Zhang ${ }^{1 *}$ and Kangsen Mai ${ }^{1}$ \\ ${ }^{1}$ The Key Laboratory of Aquaculture Nutrition and Feeds, Ministry of Agriculture, The Key Laboratory of Mariculture, Ministry \\ of Education, Ocean University of China, Qingdao 266003, People's Republic of China \\ ${ }^{2}$ Guangxi Institute of Oceanology, Key Laboratory of Marine Biotechnology of Guangxi, Beihai 536000, People's Republic of China
}

(Submitted 7 June 2017 - Final revision received 22 November 2017 - Accepted 4 December 2017)

\section{Abstract}

A previous study showed that flesh quality of large yellow croaker (LYC) was improved by feeding dietary hydroxyproline (Hyp, $0 \cdot 69 \%$ ). The aim of the present study was to explore the underlying mechanisms using transcriptomics and metabolomics analysis. The metabolomics analysis showed that muscle metabolite profiles could be clearly separated between the basal diet and Hyp supplementation diet. Metabolites including betaine, Hyp, lactate, glucose-6-phosphate, trimethylamine $N$-oxide, taurine, creatine, inosine monophosphate, histamine and serine made significant contribution to the separation. Compared with the control diet, the transcriptomics analysis identified a total of 334 different expressed genes, of which 298 genes were up-regulated and thirty-six genes were down-regulated in the Hyp supplementation group. The altered genes of the Hyp supplementation group were involved in collagen metabolism, lipid metabolism and energy metabolism. The integrated results revealed that the increased muscle collagen content in the Hyp supplementation diet was partly because of its enhancement of biosynthesis and the reduction of degradation. The improvement of muscle quality by dietary Hyp supplementation could also be related to a good utilisation of glucose through enhancement of glycolysis. It was concluded that dietary Hyp supplementation could improve flesh quality because of comprehensive metabolism changes including elevated collagen content, glycolysis, lipid metabolism and flesh flavour of LYC. The present study provided a novel strategy to understand the underlying molecular mechanism of flesh quality of LYC fed diet with Hyp supplementation.

Key words: Flesh quality: Hydroxyproline: Large yellow croaker: Metabolomics: Transcriptomics

Large yellow croaker Larimichthys crocea is popularly farmed in southern China. It has become the most large-scale mariculture fish species in China with a production of more than 148616 metric tons in $2015^{(1)}$. Along with the increasing production, quality of the farmed large yellow croaker is also receiving increasing concerns, such as skin colour and muscle texture.

Hydroxyproline (Hyp) is a substrate for the synthesis of glycine, pyruvate and glucose, as well as scavenging oxidants and regulating redox state of cells ${ }^{(2-4)}$. It is a conditionally essential amino acid in aquatic animals ${ }^{(5)}$. Meanwhile, 4-hydroxyproline is essential for intramolecular hydrogen bonds, thus contributing to the thermal stability of the triple helical domain, the integrity of the monomer and collagen fibril $^{(6)}$. In connective tissue of fresh fish muscle, collagen plays a vital role in maintaining fillet integrity and muscle cohesiveness ${ }^{(7)}$. Collagen content in muscle could be elevated when fish are fed dietary Hyp supplementation ${ }^{(8,9)}$. Moreover, dietary
Hyp could significantly enhance fish flesh quality in turbot Scophthalmus maximus L. ${ }^{(9)}$ and salmon Salmo salar L. ${ }^{(10)}$. However, it is unclear regarding the underlying molecular mechanism and metabolism variation in vivo of fish flesh quality caused by Hyp supplementation in the diet.

Transcriptomics can enable high-throughput screening of thousands of expressed genes in a given tissue, which is highly efficient and in-depth to study the development of complex phenotypic traits affected by genes ${ }^{(11)}$. Larsson et al ${ }^{(12,13)}$ used transcriptomics analysis to observe a variety of potential causes of fillet firmness, and revealed a strong positive correlation between firmness and the gene expression of Atlantic salmon (Salmo salar L.). Meanwhile, metabolomics is derived from the profiling study of metabolites, which are not merely end products of gene expression but also forming part of the regulatory system in an integrated manner ${ }^{(14)}$. Metabolomics is a powerful tool to detect the changes in metabolite content of fish treated under different conditions in quality assessment via the

Abbreviations: B-D, basal diet; H-D, Hyp supplementation diet; Hyp, hydroxyproline; TMA, trimethylamine.

* Corresponding author: W. Zhang, fax +865328203 2145, email wzhang@ouc.edu.cn 
multivariate analysis of metabolic profiles by ${ }^{1} \mathrm{H}-\mathrm{NMR}$ spectra ${ }^{(15)}$. The combination of transcriptomics and metabolomics profiling technologies has been successfully used in analyses for drug development ${ }^{(16)}$, disease diagnosis ${ }^{(17,18)}$ and nutrition health $^{(19,20)}$. Multi-omics technology was used to assess diverse data sets at different biological levels to exploit the full potential of such information for a holistic improvement of production performance, disease resistance and welfare in animals ${ }^{(21)}$. Also, the multi-omics method was used to explore the molecular mechanism and metabolism associated with phenotypic characteristics in fish muscle quality.

A previous study found that dietary Hyp significantly improved flesh quality of large yellow croaker ${ }^{(22)}$. On the basis of the total Hyp content in muscle, the optimal dietary Hyp content for large yellow croaker was estimated to be $0.61 \%$. To elucidate the underlying molecular mechanism of flesh quality improvement by dietary Hyp, in the present study the comprehensive analysis of gene expression with RNA sequence and metabolomics profile with ${ }^{1} \mathrm{H}-\mathrm{NMR}$ was used in muscle of large yellow croaker.

\section{Methods}

\section{Feeding trial and sampling}

The basal diet (B-D) was formulated to contain a combination of fish meal, maize gluten meal, soyabean meal and beer yeast as the intact protein sources. On the basis of the B-D, the Hyp supplementation diet (H-D) was made by adding $0.6 \%$ Hyp to the diet. The formula and the proximate compositions of the two experimental diets are listed in Table 1.

Large yellow croaker juveniles were obtained from a commercial farm in Ningbo, China. Before the start of the feeding trial, juveniles were reared in floating sea cages $(3.0 \times 3.0 \times 3.0 \mathrm{~m})$ for 2 weeks to acclimatise to the experimental diets and conditions. After that, fish of similar sizes (initial body weight: 189.87 (SEM 0.89) g) were randomly distributed into six sea cages $(1.5 \times 1.5 \times 2.0 \mathrm{~m})$ for two groups, and each cage was stocked with thirty-eight fish. Each group was assigned to triplicate cages, and fed one of the two experimental diets. The feeding trial lasted for $82 \mathrm{~d}$ in the sea of Xiangshan Harbour of Ningbo, Zhejiang Province, China. All the procedures were strictly carried out in accordance with the Regulations of the Experimental Animal Ethics Committee of Ocean University of China and in compliance with regulations by Institutional Animal Care and Use Committee.

\section{Sample collection}

At the end of the feeding trial, fish were fasted for $24 \mathrm{~h}$ and anaesthetised with tricaine methanesulphonate (MS-222, 1:10000) (purity 99\%, Shanghai Reagent) before sampling. In total, six fish per cage were sampled; three of them were mixed and considered as one replicated. One side of the dorsal fillet, which is above the lateral line and anterior to the dorsal fin, was collected into sterile tubes and frozen in liquid $\mathrm{N}_{2}$, and then stored at $-80^{\circ} \mathrm{C}$. Samples in liquid $\mathrm{N}_{2}$ were ground into a powder with liquid $\mathrm{N}_{2}$. The powder was sampled into a sterile tube and freeze-dried and used for ${ }^{1} \mathrm{H}-\mathrm{NMR}$ analysis.
Table 1. Formulation of the experimental diets (\%DM)

\begin{tabular}{|c|c|c|}
\hline \multirow[b]{2}{*}{ Ingredients } & \multicolumn{2}{|c|}{ Dietary Hyp levels } \\
\hline & $B-D$ & $H-D$ \\
\hline Fish meal* & $25 \cdot 0$ & $25 \cdot 0$ \\
\hline Soyabean meal* & $25 \cdot 0$ & $25 \cdot 0$ \\
\hline Wheat meal ${ }^{*}$ & $26 \cdot 0$ & $26 \cdot 0$ \\
\hline Fish oil & $6 \cdot 0$ & $6 \cdot 0$ \\
\hline Soyabean lecithin & 2.5 & 2.5 \\
\hline Mineral premix $†$ & $2 \cdot 0$ & 2.0 \\
\hline Vitamin premix $\ddagger$ & $2 \cdot 0$ & $2 \cdot 0$ \\
\hline Choline chloride & 0.2 & 0.2 \\
\hline Attractant§ & 1.5 & 1.5 \\
\hline Mould inhibitorll & 0.1 & 0.1 \\
\hline Ethoxyquine & 0.05 & 0.05 \\
\hline Amino acid premix $\mathbb{\|}$ & $5 \cdot 70$ & $5 \cdot 70$ \\
\hline Microcrystalline cellulose & $3 \cdot 15$ & $3 \cdot 15$ \\
\hline Alanine & 0.8 & 0.2 \\
\hline Hydroxyproline & 0 & 0.6 \\
\hline Total & $100 \cdot 0$ & $100 \cdot 0$ \\
\hline \multicolumn{3}{|l|}{ Proximate analysis } \\
\hline Crude protein & 43.56 & 43.42 \\
\hline Crude lipid & $12 \cdot 82$ & $12 \cdot 34$ \\
\hline Moisture (\% wet weight) & $5 \cdot 32$ & $5 \cdot 36$ \\
\hline Hydroxyproline & 0.17 & 0.69 \\
\hline
\end{tabular}

Hyp, hydroxyproline; B-D, basal diet; H-D, Hyp supplementation diet.

*All of these ingredients were supplied by Qingdao Great Seven Biotechnology Co., Ltd. Fish meal, crude protein: $74.31 \%$, crude lipid: $8.98 \%$; soyabean meal, crude protein: $57.40 \%$, crude lipid: $1.70 \%$; wheat meal, crude protein: $17.39 \%$, crude lipid: $1.47 \%$.

† Mineral premix (mg/kg diet): $\mathrm{Na}_{2} \mathrm{SeO}_{3}$ (1\%), 20, $\mathrm{Ca}\left(\mathrm{IO}_{3}\right)_{2} \cdot 6 \mathrm{H}_{2} \mathrm{O}$ (1\%), 60; $\mathrm{CoCl}_{2} \cdot 6 \mathrm{H}_{2} \mathrm{O}(1 \%), 50 ; \mathrm{CuSO}_{4} \cdot 5 \mathrm{H}_{2} \mathrm{O}, 10 ; \mathrm{FeSO}_{4} \cdot \mathrm{H}_{2} \mathrm{O}, 80 ; \mathrm{ZnSO}_{4} \cdot \mathrm{H}_{2} \mathrm{O}, 50$; $\mathrm{MnSO}_{4} \cdot \mathrm{H}_{2} \mathrm{O}, 45 ; \mathrm{MgSO}_{4} .7 \mathrm{H}_{2} \mathrm{O}, 1200 ; \mathrm{Ca}\left(\mathrm{H}_{2} \mathrm{PO}_{4}\right)_{2} \cdot \mathrm{H}_{2} \mathrm{O}, 10000$; microcrystalline cellulose, 8485 .

¥ Vitamin premix (mg/kg diet): thiamine, $25 \mathrm{~g}$; riboflavin, 45 ; pyridoxine $\mathrm{HCl}, 20$; vitamin $\mathrm{B}_{12}, 10$; vitamin $\mathrm{K}_{3}, 10$; inositol, 800; pantothenic acid, 60; niacin acid, 200; folic acid, 20; biotin, $\mathrm{Ca}\left(\mathrm{IO}_{3}\right)_{2}, 60$; retinyl acetate, 32; cholecalciferol, 5; a-tocopherol, 240; ascorbic acid, 2000; wheat middlings, 16473.

$\S$ Glycine:betaine $=1: 2$.

\| $50 \%$ calcium propionic acid and $50 \%$ fumaric acid.

II Lysine- $\mathrm{H}_{2} \mathrm{SO}_{4}$, DL-methionine, L-threonine, L-arginine, L-isoleucine, L-leucine, L-valine and L-phenylalanine.

The sample power was also used for transcriptomic assay and gene expression validation.

\section{Transcriptomic assay}

Total RNA from muscle samples was isolated by Trizol $^{\circledR}$ Reagent. The quality and integrity of RNA were examined by agarose gel electrophoresis and 2100 Bioanalyzer. The qualified RNA was treated with DNase $(5 \mathrm{U} / \mu \mathrm{l})$ (Takara) at $37^{\circ} \mathrm{C}$ for $30 \mathrm{~min}$. The DNase-treated RNA was purified by Dynabeads ${ }^{\circledR}$ Oligo (dT) 25 (Life Technologies): RNA solution is diluted to $100 \mu \mathrm{l}$ and mixed with $100 \mu \mathrm{l}$ of binding buffer. The mixture was incubated at $65^{\circ} \mathrm{C}$ for $2 \mathrm{~min}$, and then cooling on the ice rapidly. After cooling, $100 \mu \mathrm{l}$ of pre-washed beads were added and mixed at room temperature for $5 \mathrm{~min}$, and then placed on the Magnetic Grate (Life Technologies) for separation for 1-2 min. The supernatant was washed twice with $200 \mu$ of Washing Buffer B followed by the addition of $15 \mu \mathrm{l}$ of cooled $10 \mathrm{~mm}$ Tris$\mathrm{HCl}$ resuspended beads, and incubated at $75-80^{\circ} \mathrm{C}$ for $2 \mathrm{~min}$, and then placed on the Magnetic Grate for 1-2 min, immediately. The supernatants were collected to obtain the purified mRNA. The library was prepared by NEBNext ${ }^{\circledR}$ Ultra $^{\text {TM }}$ RNA Library Prep Kit for Illumina (NEB, American) according to the 
manufacturer's instructions. Random Primers and First Strand Synthesis Reaction Buffer $(5 \times)$ were added to 100 ng of purified mRNA. The mix was incubated at $94^{\circ} \mathrm{C}$ for $15 \mathrm{~min}$ and then immediately placed in ice to break in the mRNA. Murine RNase Inhibitor and ProtoScript II RT were added for the first-strand synthesis, and the reaction procedure was listed as $25^{\circ} \mathrm{C}$ for $10 \mathrm{~min}, 42^{\circ} \mathrm{C}$ for $50 \mathrm{~min}$ and $70^{\circ} \mathrm{C}$ for $15 \mathrm{~min}$. Second-Strand Synthesis Reaction Buffer $(10 \times)$ and Second-Strand Synthesis Enzyme Mix were added and incubated at $16^{\circ} \mathrm{C}$ for $1 \mathrm{~h}$ to synthesise the second strand. After adding 1.8 times volume AMPure XP Beads (Agencourt) to purify the reagent obtained from the last step, the End Repair Reaction Buffer (10X) and End Prep Enzyme Mix were added and incubated at $20^{\circ} \mathrm{C}$ for $30 \mathrm{~min}$, and then $65^{\circ} \mathrm{C}$ for $30 \mathrm{~min}$ for end repairing. NEBNext Adaptor and Blunt/TA Ligase Master Mix were added and incubated at $20^{\circ} \mathrm{C}$ for $15 \mathrm{~min}$ for adaptor linkage, followed by the addition of USER Enzyme at $37^{\circ} \mathrm{C}$ for $15 \mathrm{~min}$ to excise $\mathrm{U}$ in adaptor. After adding and mixing 0.6 times volume of AMPure XP Beads, the mixture was placed on the Magnetic Grate for $5 \mathrm{~min}$ after allowing it to stand for $5 \mathrm{~min}$. The supernatant was collected, and then 0.25 times volume of AMPure XP Beads was added for purification. The 300-500-bp-length ligated cDNA was made. Universal PCR Primer and Index (X) Primer were used for PCR amplification. After preheating at $98^{\circ} \mathrm{C}$ for $10 \mathrm{~s}, 12-15$ cycles of amplification were performed using a cycle profile as denaturation at $98^{\circ} \mathrm{C}$ for $10 \mathrm{~s}$, annealing at $65^{\circ} \mathrm{C}$ for $30 \mathrm{~s}$ and extension at $72^{\circ} \mathrm{C}$ for $30 \mathrm{~s}$. The reagent was purified by one times volume AMPure XP Beads and the library for sequencing was obtained. The $2 \%$ agarose gel electrophoresis, Qubit quantification and High-sensitivity DNA chip were used to obtain qualified cDNA library. The sequencing of cDNA in the library was performed on Illumina Hiseq ${ }^{\mathrm{TM}} 2500$ after using TruSeq PE Cluster Kit (Illumina) performing cluster generation on cBot.

\section{Gene expression validation}

Genes obtained from the transcriptomic assay were validated and quantified by quantitative real-time PCR (qRT-PCR). The primers used for qRT-PCR are listed in Table 2. Total RNA used in RT-PCR analysis was isolated with Trizol reagent (Invitrogen) from the same samples as that in transcriptomic assay. The qRT-PCR was performed using SYBR Green as the fluorescent dye according to the manufacturer's protocol (Takara). Each sample was run in triplicate. The mRNA expression levels were normalised by the reference gens and calculated using the comparative cycle threshold $\left(C_{t}\right)$ values expressed as $2^{-\Delta \Delta C_{t}}$. GraphPad Prism 7.0 was used for the interpretation and analysis of data.

\section{${ }^{1} \mathrm{H}-\mathrm{NMR}$ analysis}

Sample preparation for ${ }^{1} \mathrm{H}-\mathrm{NMR}$ analysis. In brief, lyophilised muscle powder $(50 \mathrm{mg}$ ) was weighed into a centrifuge tube and suspended in $1 \mathrm{ml}$ of purified water. The tube was then sonicated (VCX-130; Sonics \& Materials, Inc.) on ice. The ultrasonic extraction was repeated eight times for 4 and 3-s intervals with the cycling programme. Samples were centrifuged (Eppendorf Centrifuge 5415R) at $13000 \mathrm{rpm}$ for $15 \mathrm{~min}$ at $4^{\circ} \mathrm{C}$. The aqueous layer was collected and filtered by amicon Ultra 0.5-ml centrifugal filters with 3-kDa cut-off (Millipore). The
Table 2. Primers for relative quantitative real-time PCR

\begin{tabular}{|c|c|}
\hline Gene & Primer sequence $\left(5^{\prime}-3^{\prime}\right)$ \\
\hline$\beta$-Actin & $\begin{array}{l}\text { F: GACCTGACAGACTACCTCATG } \\
\text { R: AGTTGAAGGTGGTCTCGTGGA }\end{array}$ \\
\hline col1a1 & $\begin{array}{l}\text { F: CAACAGCCGCTTCACATACA } \\
\text { R: ACAGACGGGTCCAACTTCAA }\end{array}$ \\
\hline col6a1 & $\begin{array}{l}\text { F: GGATACGTGTTGCTCCTTCG } \\
\text { R: TTTCACCCTTTTCTCCTCTGC }\end{array}$ \\
\hline col5a2 & $\begin{array}{l}\text { F: TGTGACGAAATCCAGTGCG } \\
\text { R: GGTGTTGGTGGTGCTTGTATC }\end{array}$ \\
\hline timp2 & $\begin{array}{l}\text { F: GTGATTAGAGCAAAGGTGGTGG } \\
\text { R: CATTGGCATCCAGGGTAACA }\end{array}$ \\
\hline gatm & $\begin{array}{l}\text { F: TCTCATTCAAGGACCCCAAC } \\
\text { R: GAATCAGAGGTGTCGGAGGTT }\end{array}$ \\
\hline sdh & $\begin{array}{l}\text { F: CACCAACATTAGCAAACCAACT } \\
\text { R: CATTCATACAGCCCGTCCA }\end{array}$ \\
\hline bhmt & $\begin{array}{l}\text { F: GAGATGGCACCTGTCAAGAAG } \\
\text { R: CCAGAGCAAACACGAAACCT }\end{array}$ \\
\hline pfkm & $\begin{array}{l}\text { F: TTGGTGGATTTGAGGCTTATG } \\
\text { R: TAGAGGTGATGGTGTTGAGGG }\end{array}$ \\
\hline scd1 & $\begin{array}{l}\text { F: GGTACTTCTGGGGTGAATCCT } \\
\text { R: ATGGCACTGAAAGCAACTAATG }\end{array}$ \\
\hline p4ha1 & $\begin{array}{l}\text { F: CCATACATTGTCCGCTACCTT } \\
\text { R: GCCTTAATCGTGGCTTTGC }\end{array}$ \\
\hline
\end{tabular}

filtrate was centrifuged at $13000 \mathrm{rpm}$ for $30 \mathrm{~min}$ at $4^{\circ} \mathrm{C}$. The top $450-\mu \mathrm{l}$ aqueous layer was collected and transferred to a clean 2-ml centrifuge tube. A 50- $\mu$ 1 2,2-dimethyl-2-silapentane-5sulphonate standard solution (Anachro) was added in the tube. Samples were vortex-shocked well for $10 \mathrm{~s}$ and centrifuged at $13000 \mathrm{rpm}$ for $2 \mathrm{~min}$ at $4^{\circ} \mathrm{C}$. The $480 \mu \mathrm{l}$ of supernatant was transferred to ${ }^{1} \mathrm{H}-\mathrm{NMR}$ tube for analysis.

${ }^{1} \mathrm{H}$-NMR spectroscopy. Spectra were collected using a Bruker AV III $600 \mathrm{MHz}$ spectrometer equipped with an inverse cryoprobe operating at a proton NMR frequency of $600 \cdot 13 \mathrm{MHz}$. The first increment of a 2D-1H, ${ }^{1} \mathrm{H}-\mathrm{NOESY}$ pulse sequence was used for the acquisition of ${ }^{1} \mathrm{H}-\mathrm{NMR}$ data and for suppressing the solvent signal. Spectra were collected at $25^{\circ} \mathrm{C}$, with a total of 180 scans over a period of about $20 \mathrm{~min}$.

Quantification of the metabolites. The collected free induction decay signal was automatically zero-filled and fouriertransformed in processing module in Chenomx NMR Suite 7.7 (Chenomx Inc.). The data were then carefully phased and corrected to baseline in the Chenomx Processor. All the spectra were referenced for the quantification to the internal standard. The quantification was performed by comparing the integral of a known reference signal (DSS-d6) with the signals derived from a library of compounds containing chemical shifts. The identifiable metabolites were chosen for quantification by matching to the Chenomx Library. All concentrations of metabolites were collected and normalised by weight across all parallel samples before being used in the later multivariable analysis.

\section{Data analysis}

The chemometric analysis for metabolites was performed in the $\mathrm{R}$ software environment using the PLS package ${ }^{(23)}$. Plots were made using the ggplot2 package ${ }^{(24)}$. The variable importance in 
the projection (VIP) values and corresponding loadings for partial least squares (PLS) models were applied to identify the variable. If VIP is greater than 1 , this variable was considered a very important variable for the model and selected as candidate biomarker variables ${ }^{(25)}$. It was also considered to be significant.

For gene expression determination, reads per kilobase of gene per million mapped reads (RPKM) was performed as a standard calculation, which quantifies gene expression from RNA sequencing data by normalising for total read length and the number of sequencing reads ${ }^{(26)}$. The gene expression abundance between the two samples was calculated using the MAplot-based method with the random sampling model in the DEGseq package ${ }^{(27)}$. The false discovery rate (FDR) was used to determine the $P$ value threshold. We have used stringent criteria to identify differentially expressed genes (DEG) at FDR $<0.001$, $\mid \log _{2}$ fold change $>1, q$ value $(P$ value was adjusted using Benjamini) $<0.001$ and RPKM $>20$ for at least one sample. A search for enriched gene ontology (GO) classes and KEGG pathways of DEG was performed. An enrichment score threshold of $q$ value $<0.05$ was selected for statistical significance.

\section{Results}

\section{Gene expression profiling by RNA sequence}

According to the screening criteria $\left(q<0 \cdot 001\right.$, RPKM $>20, \log _{2}$ (fold change) $>1$ ), a total of 334 genes were identified as DEG, of which 112 genes were not annotated in the B-D group and H-D group. Meanwhile, 298 genes and thirty-six of these DEG were significantly up-regulated and down-regulated, respectively, in the H-D group. The results of gene expression validation from qRT-PCR are shown in Fig. 1. The expression levels of ten genes selected were up-regulated in the B-D diet compared with the H-D diet, which showed a high consistent trend from the results of transcriptomic assay.

The GO and KEGG classified analysis of DEG is shown in Table 3. These genes participate in the pathway of antigen processing and presentation, PPAR signalling pathway, protein digestion and absorption. Meanwhile, enrichment of DEG was observed in extracellular matrix-receptor interaction, extracellular region part, extracellular region, macromolecular complex, organelle part and structural molecule activity. Genes in the group that was supplemented with Hyp showed sizeable

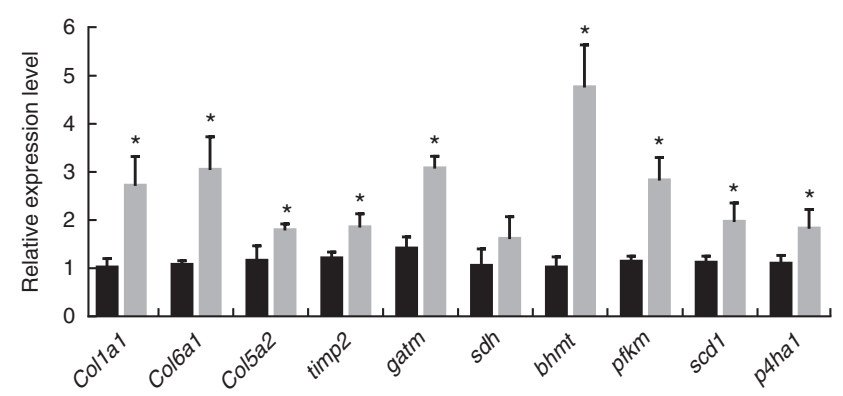

Fig. 1. Quantitative real-time PCR of selected genes from the transcriptomic profile. The gene expression level is the relative average $2^{-\Delta \Delta C t}$ as normalised to the housekeeping gene $\beta$-actin. Values are means with standard errors represented by vertical bars, and statistical analysis was done by unpaired $t$ test ( $\left.{ }^{\star} P<0.05\right)$. ( $\square$ B-D, basal diet; $(\square) \mathrm{H}-\mathrm{D}$, Hyp supplementation diet. and mainly increased expression. Examples are given in Table 4. A $\log _{2}$ (fold change) value greater than 1 indicated an up-regulation in the H-D group compared with the B-D group, and the ones less than -1 indicated a down-regulation in the H-D group compared with the B-D group. In comparison with the B-D group, gene expression of betaine-homocysteine S-methyltransferase (bhmt) involved in glycine, serine and threonine metabolism, cysteine and methionine metabolism in the H-D group was up-regulated. Other up-regulation of genes involved in amino acid metabolism including glycine amidinotransferase (gatm), glycyl-tRNA synthetase (gars) and aminoacylase 1 (acy1) were observed in the H-D group. Expression levels of aconitate hydratase (aco), ATP-dependent 6-phosphofructokinase, muscle type-like (pfkm), fructosebisphosphate aldolase A (aldoa), glyceraldehyde-3-phosphate dehydrogenase (gapdh) and succinate debydrogenase ( $s d b)$ involved in glucose degradation of carbohydrate catabolism were increased in the H-D group. Several other genes, which were involved in collagen gene expression, biosynthesis and degradation, were up-regulated in the H-D group. These genes included collagen $\alpha-1(I)$ chain-like (col1a1), collagen $\alpha-2(V)$ chain-like (col5a2), collagen, type VI, a (col6a), protein disulphide-isomerase A1 (pidia1), prolyl 4-hydroxylase subunit $\alpha-1$ isoform X2 (p4ha1), lysyl oxidase (lox) and tissue inhibitor of metalloproteinase 2 (timp2). The gene expression of col1a1 showed the greatest up-regulation $\left(\log _{2}\right.$ (fold change) $=5 \cdot 18$ ) Up-regulated genes involved in lipid metabolism included binding protein (fabp3), fatty acid-binding protein $2 a$ (fabp2), stearoyl-CoA desaturase ( $\Delta-9$ desaturase) (scd1), lipoprotein lipase (lpl), diacylglycerol O-acyltransferase 2 (dgat) and long-chain fatty acid transport protein 1-like (fatp1). These genes also participated in the PPAR signalling pathway. The genes involved in energy metabolism and immune system were observed. Muscle fibre-related genes including in myosin beavy chain (mby), myosin light polypeptide 6 (mylo) and calpain-3 (capn3) were up-regulated.

Table 3. Enrichment terms of gene ontology (GO) and the KEGG pathway of differentially expressed genes in muscle of large yellow croaker fed dietary hydroxyproline supplementation

\begin{tabular}{llcc}
\hline & $\begin{array}{l}\text { GO/KEGG } \\
\text { number }\end{array}$ & Genes & $q$ Value \\
Term & ko04612 & 9 & 0.000000 \\
\hline Antigen processing and presentation & ko04260 & 7 & 0.000162 \\
Cardiac muscle contraction & ko03320 & 6 & 0.000746 \\
PPAR signalling pathway & ko04261 & 6 & 0.021327 \\
$\begin{array}{l}\text { Adrenergic signalling in } \\
\quad \text { cardiomyocytes }\end{array}$ & & & \\
Protein digestion and absorption & ko04974 & 4 & 0.048195 \\
Intestinal immune network for IgA & ko04672 & 2 & 0.048195 \\
$\quad$ production & & & \\
Extracellular matrix-receptor & ko04512 & 4 & 0.048195 \\
$\quad$ interaction & & & \\
Extracellular region part & GO:0044421 & 13 & 0.022017 \\
Extracellular region & GO:0005576 & 22 & 0.006266 \\
Macromolecular complex & GO:0032991 & 52 & 0.000037 \\
Organelle part & GO:0044422 & 64 & 0.006529 \\
Cellular component biogenesis & GO:0044085 & 18 & 0.006529 \\
Establishment of localisation & GO:0051234 & 40 & 0.007237 \\
Localisation & GO:0051179 & 41 & 0.038536 \\
Structural molecule activity & GO:0005198 & 26 & 0.000000 \\
\hline
\end{tabular}


Table 4. Differentially expressed genes in muscle of larger yellow croaker fed dietary hydroxyproline supplementation

\begin{tabular}{|c|c|c|}
\hline Gene & Description & $\begin{array}{c}\log _{2} \text { (fold } \\
\text { change) } \mathrm{H}-\mathrm{D} / \mathrm{B}-\mathrm{D}\end{array}$ \\
\hline Aat & ADP/ATP translocase 2 like & 1.79 \\
\hline Aco & Aconitate hydratase, mitochondrial & 1.32 \\
\hline Acs/1 & $\begin{array}{l}\text { Long-chain fatty acid-CoA ligase } \\
1 \text { isoform X2 }\end{array}$ & $2 \cdot 52$ \\
\hline Acta1 & Actin, $a$ skeletal muscle & 1.36 \\
\hline Acy 1 & Aminoacylase 1 & $1 \cdot 17$ \\
\hline Aldoa & Fructose-bisphosphate aldolase A & $2 \cdot 35$ \\
\hline Arf1 & ADP-ribosylation factor 1 like & 1.25 \\
\hline Atp2a1 & $\begin{array}{l}\text { Sarcoplasmic/endoplasmic reticulum } \\
\text { calcium ATPase } 1 \text { like }\end{array}$ & $2 \cdot 00$ \\
\hline Atp6 & ATP synthase F0 subunit 6 & $1 \cdot 27$ \\
\hline$B 2 m$ & $\beta$-2-microglobulin precursor & 1.46 \\
\hline Bhmt & Betaine-homocysteine $S$-methyltransferase 1 & $2 \cdot 52$ \\
\hline CA2 & Carbonic anhydrase 2 & 1.75 \\
\hline Capn3 & Calpain-3 & $2 \cdot 10$ \\
\hline$C d 74$ & $\begin{array}{l}\text { Major histocompatibility complex class II } \\
\text { antigen-associated invariant chain }\end{array}$ & $2 \cdot 24$ \\
\hline Cd9912 & CD99 antigen-like protein 2 like & 1.04 \\
\hline Cfl2 & Cofilin-2 & 1.76 \\
\hline Col1a1 & Type 1 collagen a 1 & $5 \cdot 18$ \\
\hline Col5a2 & Collagen $a-2(\mathrm{~V})$ chain like & $2 \cdot 69$ \\
\hline Col6a1 & Collagen, type $\mathrm{VI}, a 1$ & $2 \cdot 61$ \\
\hline Суb5r3 & NADH-cytochrome b5 reductase 3 like & 1.08 \\
\hline Cytb & Cytochrome b & 1.02 \\
\hline Den & Decorin & $2 \cdot 12$ \\
\hline Dgat2 & Diacylglycerol $O$-acyltransferase 2 & 4.70 \\
\hline Fabp2 & Fatty acid-binding protein $2 a$ & 1.29 \\
\hline Fabp3 & Muscle fatty acid-binding protein 3 & 1.50 \\
\hline Fatp1 & Long-chain fatty acid transport protein 1 like & 1.03 \\
\hline Fbxo32 & F-box protein 32 like & -1.48 \\
\hline Gapdh & Glyceraldehyde-3-phosphate dehydrogenase & 1.25 \\
\hline Gars & Glycyl-tRNA synthetase & 1.28 \\
\hline Gatm & Glycine amidinotransferase & $1 \cdot 11$ \\
\hline Gst & Glutathione $S$-transferase & 1.23 \\
\hline Hsp90b & Heat-shock protein $90-\beta$ & 1.52 \\
\hline Hspa1-8 & Heat-shock $70-k D a$ protein $1 / 8$ & 1.07 \\
\hline Loxl2a & Lysyl oxidase homolog 2A like & 1.55 \\
\hline Loxl2b & Lysyl oxidase homolog 2B like & $2 \cdot 23$ \\
\hline Loxl3 & Lysyl oxidase homolog 3 isoform X2 & 1.55 \\
\hline Lpl & Lipoprotein lipase & 2.49 \\
\hline Mhc1 & Major histocompatibility complex, class I & 2.03 \\
\hline Mhc2 & Major histocompatibility complex, class II & $2 \cdot 20$ \\
\hline Mhy & Myosin heavy chain & $2 \cdot 22$ \\
\hline Myl4 & Myosin light chain 4 like & 2.05 \\
\hline Myl6 & Myosin light polypeptide 6 & $2 \cdot 40$ \\
\hline Ndufa4 & $\begin{array}{l}\text { NADH dehydrogenase (ubiquinone) } \\
1 \text { a Subcomplex subunit } 4\end{array}$ & 1.43 \\
\hline P4ha1 & $\begin{array}{l}\text { Prolyl 4-hydroxylase subunit } \\
\quad a-1 \text { isoform X2 }\end{array}$ & 1.68 \\
\hline Pcolce2 & $\begin{array}{l}\text { Procollagen C-endopeptidase enhancer } \\
2 \text { precursor }\end{array}$ & 1.94 \\
\hline Pdia1 & Protein disulphide-isomerase A1 & 1.27 \\
\hline Pdia3 & $\begin{array}{l}\text { Protein disulphide isomerase family A, member } \\
3\end{array}$ & 1.46 \\
\hline Pfkm & $\begin{array}{l}\text { ATP-dependent 6-phosphofructokinase, } \\
\text { muscle type like }\end{array}$ & $1 \cdot 32$ \\
\hline Scd1 & Stearoyl-CoA desaturase ( $\triangle-9$ desaturase) & 3.83 \\
\hline$S d h$ & Succinate dehydrogenase & 1.03 \\
\hline Smyhc2 & Slow myosin heavy chain 2 & $2 \cdot 26$ \\
\hline Ssr1 & Translocon-associated protein subunit $a$ & 1.48 \\
\hline Timp2 & Tissue inhibitor of metalloproteinase 2 & $3 \cdot 12$ \\
\hline Tkt & Transketolase & $2 \cdot 12$ \\
\hline Tnni1 & Troponin I, slow skeletal muscle like & 1.73 \\
\hline Tpm1 & Tropomyosin $a-1$ chain-like isoform $\mathrm{X} 2$ & 1.49 \\
\hline TTN & Titin & 1.59 \\
\hline
\end{tabular}

B-D, basal diet; H-D, Hyp supplementation diet.

\section{Metabolite profiling by ${ }^{1} \mathrm{H}-\mathrm{NMR}$ spectroscopy}

A typical ${ }^{1} \mathrm{H}$-NMR spectrum in the present study is shown in Fig. 2. A total of sixty-two metabolites were identified in the spectra for the muscle samples of large yellow croaker. Amino acids and their derivatives, organic acids, sugars and nucleic acid components were detected. A partial least squares discriminant analysis (PLS-DA) classification model was constructed to evaluate the metabolic differences of muscle tissues between the two groups (Fig. 3). The score plot (Fig. 3(a)) shows that the B-D group and the H-D group are clearly distinct from each other. The corresponding loading plot (Fig. 3(b)) helps the visual selection of biomarkers. It indicates that the further the metabolites get away from the core, the more the contributions can be distinguished from the two groups. The metabolites with VIP above 1 (Table 5) are significant variables that contribute to distinguishing the two groups. The concentration of metabolites in the B-D diet including trans-4-hydroxy-L-proline, glucose-6phosphate, trimethylamine $\mathrm{N}$-oxide (TMAO), taurine and inosinic acid (inosine monophosphate (IMP)) were up-regulated compared with those in the group of H-D, whereas the metabolites including betaine, lactate, creatine, histamine and serine were down-regulated. These biomarker metabolites were mainly related to the pathways of collagen metabolism, amino acid metabolism, glucose metabolism and fish muscle flavour.

\section{Discussion}

In the previous study, Hyp supplementation of $0.6 \%$ in diet increased the muscle texture parameters (hardness, adhesiveness, springiness and chewiness), $\mathrm{pH}$ and lipid-holding capacity compared with the control group, which indicated an improvement of flesh quality ${ }^{(22)}$. The present study explores the possible underlying mechanism.

Higher collagen content is closely related to firmer fillet and higher muscle $\mathrm{pH}$ in sea bass, Dicentrarchus labrax L. ${ }^{(28)}$. A previous study on large yellow croaker also found that dietary supplementation of Hyp significantly increased collagen content in muscle ${ }^{(22)}$. In the present study, the expression of three collagen genes (col1a1, col5a2 and col6a) observed from the transcriptomics was up-regulated in the H-D group. Five types of collagen (types I, III, IV, V and VI) were found in the muscle of Gadus morbua ${ }^{(29)}$. Type I collagen is the most abundant and studied collagen, whereas the content of type $\mathrm{V}$ collagen is relatively less, but more reported in fish connective tissue related with flesh quality ${ }^{(30)}$. A coordinated significant variation of expression was observed in genes involved in the metabolism of collagen. Four collagen biosynthesis-related genes including pidia1, pcolce2, p4ha1 and lox observed from the transcriptomics was up-regulated in the Hyp supplementation group. The p4ha1 plays a central role in collagen synthesis. It catalyses the formation of 4-Hyp in collagens and other proteins with collagen-like sequences by the hydroxylation of proline residues in peptide linkages ${ }^{(31)}$. The lox is essential to promote cross-linking and plays a critical role in the biogenesis of connective tissue matrices, which catalyse the oxidative deamination of the $\varepsilon$-amino group of lysines and hydroxylysines in collagen and elastin ${ }^{(32)}$. Additionally, the gene of tissue inhibitor 


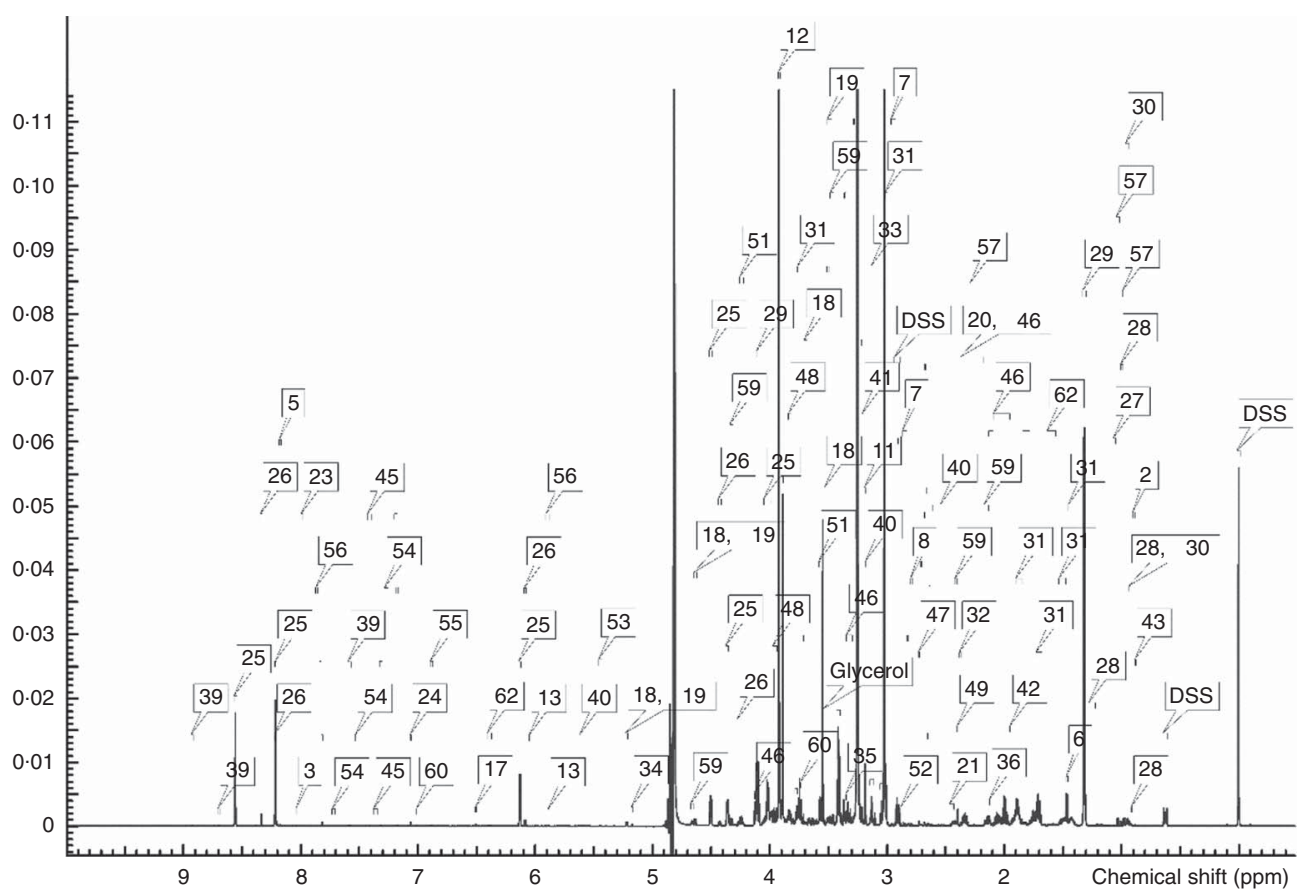

Fig. 2. A typical ${ }^{1} \mathrm{H}$-NMR spectrum obtained from muscle samples of large yellow croaker fed diet with hydroxyproline supplementation. 1, 2-aminobutyrate; 2 , 2-hydroxybutyrate; 3, 3-methylxanthine; 4, acetate; 5, adenine; 6, alanine; 7, asparagine; 8, aspartate; 9 , betaine; 10, carnitine; 11, choline; 12, creatine; 13, cytidine; 14, dimethylamine; 15, ethanol; 16, formate; 17, fumarate; 18, glucose; 19, glucose-6-phosphate; 20, glutamate; 21, glutamine; 22, glycine; 23, guanosine; 24 , histamine; 25, inosine monophosphate; 26 , inosine; 27 , isobutyrate; 28 , isoleucine; 29 , lactate; 30 , leucine; 31, lysine; 32, malate; 33 , malonate; 34, mannose; 35, methanol; 36, methionine; 37, N,N-dimethylglycine; 38, N-methylhydantoin; 39, niacinamide; 40, O-acetylcarnitine; 41, O-phosphocholine; 42, ornithine; 43 , pantothenate; 44 , trimethylamine $\mathrm{N}$-oxide; 45 , phenylalanine; 46 , proline; 47 , sarcosine; 48 , serine; 49 , succinate; 50 , taurine; 51 , threonine; 52 , trimethylamine; 53, glucose-1-phosphate; 54, tryptophan; 55, tyrosine; 56, uridine; 57, valine; 58, sn-glycero-3-phosphocholine; 59, trans-4-hydroxy-L-proline; $60, \pi$-methylhistidine; 61, 3-aminoisobutyrate; 62, citrulline; ppm, Parts per million.

of metalloproteinase has the ability to inhibit matrix metalloproteinases (MMP) ${ }^{(33)}$. In the present study, the gene expression of the timp2 observed from the transcriptomics was significantly up-regulated by the supplementation of Hyp in diet. It is hypothesised that the MMP play important roles in the degradation of collagens, thus causing the postmortem softening of fish muscle. For example, MMP-2 is a major proteinase responsible for the degradation of type $\mathrm{I}$ and $\mathrm{V}$ collagens in common carp (C. carpio), thus resulting in the softening of fish muscle during chilled storage ${ }^{(34)}$. The results of the present study suggest that dietary Hyp regulated collagen turnover in muscle partly through increasing the ability of collagen biosynthesis and suppressing the degradation of collagen in the muscle of large yellow croaker, which is in agreement with the previous study ${ }^{(22)}$. A similar study was observed in rat, where an oral intake of L-hydroxyproline had a stimulatory effect on collagen metabolism and turnover ${ }^{(35)}$.

Apart from collagen metabolism, dietary Hyp could play a role in taurine-related amino acid metabolism. The intake of certain nutrients can affect gene expression of bhmt in salmonid, such as methionine ${ }^{(36)}$. In the present study, the expression of bhmt observed from the transcriptomics showed up-regulation by dietary Hyp supplementation. The bhmt is a cytosolic enzyme that catalyses the conversion of betaine and homocysteine to dimethylglycine and methionine, respectively ${ }^{(37)}$. Methionine is a precursor for taurine biosynthesis; its degradation leads to the synthesis of taurine ${ }^{(38)}$. This mechanism is consolidated by the increased concentration of taurine observed from the metabolomics. It suggests that Hyp supplementation in fish diet could affect taurine metabolism leading to the increased concentration of taurine. Taurine plays an important role in multiple physiological processes such as the regulation of antioxidation ${ }^{(39)}$ and acting as a $\mathrm{pH}$ buffer for regulation of mitochondrial metabo$\operatorname{lism}^{(40)}$. It was also reported in mammals that taurine was involved in the control of glucose homoeostasis by regulating the expression of genes that are required for glucose-stimulated insulin secretion and that it enhances peripheral insulin sensitivity ${ }^{(41)}$. The present study showed that an increased concentration of glucose-6-phosphate observed from the metabolomics coincided with an up-regulated gene expression of glycolysis-related genes including $p f k m$, aldoa and gapdh observed from the transcriptomics in the Hyp-supplemented group, which indicated a better utilisation of carbohydrates. It is well known that teleost fish show persistent hyperglycaemia and are generally considered to be glucose intolerant ${ }^{(42)}$. The results suggest a tendency towards better utilisation of glucose when fish are fed dietary Hyp, and provide evidence that the regulation of glucose by Hyp supplementation could be partly due to the increased taurine content. The enhancement of glycolysis involved in the aerobic metabolism correlates with a firm fillet. A previous study observed that fish fed dietary Hyp supplementation had a firmer fillet ${ }^{(22)}$. The present study showed that several gene expression increased observed from the trancriptomics, such as $s d h$ (a citric acid cycle enzyme) and aco. 
(a)

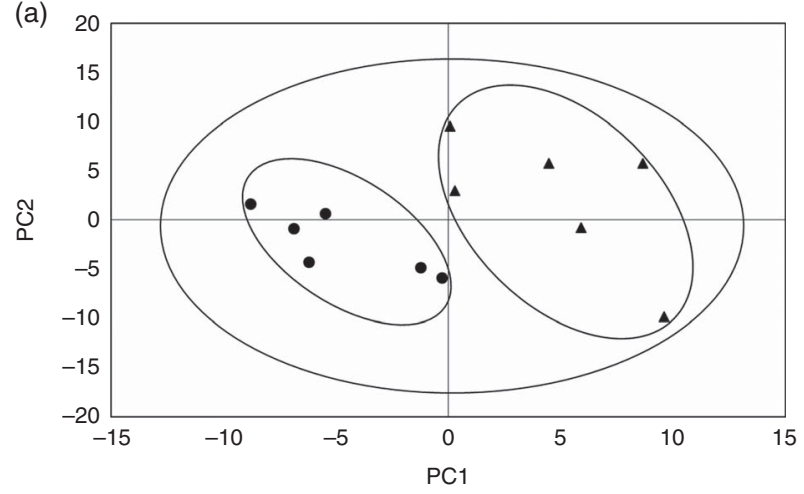

(b)

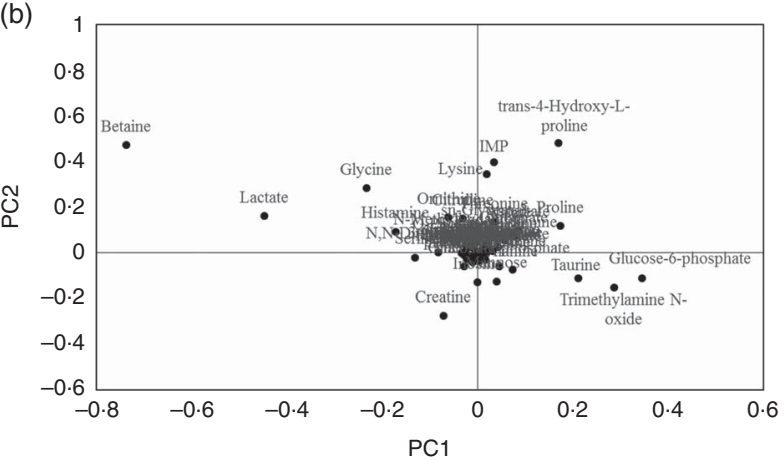

Fig. 3. The partial least squares discriminant analysis (PLS-DA) model in muscle of large yellow croaker fed dietary hydroxyproline supplementation. The components score plot in muscle of large yellow croaker fed a diet with no Hyp supplementation compared with those fed a diet with Hyp supplementation, the ellipses indicate clusters of the treatment groups in the experiment (a). Loading plot that explained the further the metabolites get away from the core, the more the contributions distinguishing two groups (b). (O) B-D, basal diet; (A) H-D, Hyp supplementation diet.

Table 5. Variable importance in projection (VIP) scores of muscle metabolites of large yellow croaker fed dietary hydroxyproline supplementation*

\begin{tabular}{lll}
\hline Metabolite & VIP & H-D/B-D \\
\hline Betaine & 4.32 & Down \\
Trans-4-hydroxy-L-proline & 3.32 & Up \\
Lactate & 2.76 & Down \\
Glucose-6-phosphate & 1.96 & Up \\
Trimethylamine N-oxide & 1.63 & Up \\
Taurine & 1.39 & Up \\
Creatine & 1.30 & Down \\
IMP (inosinic acid) & 1.29 & Up \\
Histamine & 1.20 & Down \\
Serine & 1.00 & Down \\
\hline
\end{tabular}

B-D, basal diet; H-D, Hyp supplementation diet; IMP, inosine monophosphate. * It shows the VIP >1 metabolites, which contributed to a distinction between control group and Hyp supplementation group.

These genes are vital regulators of energy metabolism regarding mitochondrial functions. This agrees with the study of Atlantic salmon that showed a higher mitochondrial metabolism with firmer fillets ${ }^{(12,13)}$. In the present study, the increased carbohydrate metabolism together with mitochondrial processes indicated an enhanced cellular aerobic respiration in the Hyp supplementation group, which could be attributed to a good energy utilisation with a firmer fillet.
The PPAR signal pathway, which is related with the lipid metabolism, was significantly enriched as observed from the transcriptomics in the present study. In this pathway, $l p l$ was up-regulated. The $l p l$ is a key enzyme in lipid metabolism that hydrolyses TAG to provide free fatty acids for cells and affects the maturation of circulating lipoproteins ${ }^{(43)}$. It is also known as an activator (and/or ligands) of the various $\operatorname{PPAR}^{(44)}$. The fabp3 is known to function as a transport protein for mitochondrial $\beta$-oxidation in muscle tissues ${ }^{(45)}$. It increased in the H-D group in the present study. The fatp1 and acyl-CoA acsl1 were also improved in the H-D group and, respectively, facilitated the transport of fatty acids across the cell membrane. Meanwhile, their esterification prevented their efflux and facilitated fatty acid uptake ${ }^{(46)}$. In addition, $s c d 1$, which plays an important role in lipogenesis, was also up-regulated. It is the rate-limiting enzyme that catalysed the conversion of saturated long-chain fatty acids into MUFA ${ }^{(47)}$. The results indicated that fish fed dietary Hyp supplementation enhanced the lipid metabolism. The up-regulated gene of lipid metabolism owing to Hyp supplementation indicates that the consumption of fat as fuel provided cellular energy, which was verified by the expression of genes involved in energy metabolism. Positive correlations with firmness were shown for genes involved in different aspects of lipid metabolism ${ }^{(12)}$. This was further confirmed with the firmer muscle texture in Hyp supplementation group in the previous study ${ }^{(22)}$.

IMP is a breakdown/degradation product of ATP. It is an important taste enhancer and used as an index of freshness quality in a wide variety of fish ${ }^{(48,49)}$. Several researches have also reported that the decrease or disappearance of IMP is correlated with the loss of fresh fish flavour in most species ${ }^{(50,51)}$. The high level of IMP in the H-D group observed from the metabolomics in the present study indicated a good flesh freshness in muscle of large yellow croaker. The level of trimethylamine (TMA) is strongly correlated to the sensory quality of $\operatorname{cod}^{(52)}$, and can be also used as an objective index to predict the fish quality in freshness ${ }^{(53)}$. Its concentration increases with the decrease of the fish freshness ${ }^{(54)}$. The TMA is secreted by the decomposition of TMAO, and its concentration increased after the fish died ${ }^{(55)}$. An inhibition of conversion from TMAO to TMA will have a better fish freshness. In the present study, although no change was found for TMA, the concentration of TMAO observed from the metabolomics increased. It suggests a tendency towards a good flesh freshness when fish is fed a diet with supplementation of Hyp.

In conclusion, combining analysis of transcriptomics and metabolomics revealed that the increased collagen content in muscle of large yellow croaker fed a diet with supplementation of Hyp was partly due to the enhancement of synthesis and the diminished degradation of collagen. The up-regulation of glycolysis coinciding with the mitochondrial processes suggests better utilisation of carbohydrates and an enhanced cellular aerobic respiration in the Hyp supplementation group. This could lead to a good energy utilisation with a firm fillet of large yellow croaker. The enhancement PPAR signalling pathway was significantly enriched by Hyp supplementation and it had stimulatory effects on improving flesh quality of large yellow croaker. Meanwhile, dietary Hyp could improve fish freshness by inhibiting conversion from TMAO to TMA. 


\section{Acknowledgements}

The authors thank the participants who contributed to this trial.

This work was financially supported by the National Natural Science Foundation of China (grant no. 31372542), and the Fundamental Research Funds for the Central Universities of Ocean University of China (grant no. 201562017).

Z. W. completed the experiment and prepared the manuscript. H. Z. and Y. Z. analysed the samples. W. Z. designed the experiment and revised the manuscript. K. M. designed the experiment.

The authors declare that there are no conflicts of interest.

\section{References}

1. Fishery Bureau, Ministry of Agriculture (2016) China Fishery Statistical Yearbook, p. 28. Beijing, China: China Agriculture Press.

2. Wu G, Bazer FW, Burghardt RC, et al. (2010) Proline and hydroxyproline metabolism: implications for animal and human nutrition. Amino Acids 40, 1053-1063.

3. Phang JM, Donald SP, Pandhare J, et al. (2008) The metabolism of proline, a stress substrate, modulates carcinogenic pathways. Amino Acids 35, 681-690.

4. Takayama T, Fujita K, Suzuki K, et al. (2003) Control of oxalate formation from L-hydroxyproline in liver mitochondria. JASN 14, 939-946.

5. Li P, Mai K, Trushenski J, et al. (2009) New developments in fish amino acid nutrition: towards functional and environmentally oriented aquafeeds. Amino Acids 37, 43-53.

6. Gelse K, Poschl E \& Aigner T (2003) Collagensstructure, function, and biosynthesis. Adv Drug Deliv Rev $\mathbf{5 5}$, $1531-1546$.

7. Aussanasuwannakul A, Slider SD, Salem M, et al. (2012) Comparison of variable-blade to Allo-Kramer shear method in assessing rainbow trout (Oncorbynchus mykiss) fillet firmness. J Food Sci 77, S335-S341.

8. Zhang K, Ai Q, Mai K, et al. (2013) Effects of dietary hydroxyproline on growth performance, body composition, hydroxyproline and collagen concentrations in tissues in relation to prolyl 4-hydroxylase $\alpha(\mathrm{I})$ gene expression of juvenile turbot, Scophthalmus maximus L. fed high plant protein diets. Aquaculture 404, 77-84.

9. Liu Y, He G, Wang Q, et al. (2014) Hydroxyproline supplementation on the performances of high plant protein source based diets in turbot (Scophthalmus maximus L.). Aquaculture 433, 476-480.

10. Albrektsen S, Sirnes E, Aksnes A, et al. (2010) Impacts of dietary hydroxyproline on growth, muscle firmness, collagen and PYD cross-links formation in Atlantic salmon (Salmo salar). Program and Abstracts of the 14th International Symposium on Fish Nutrition and Feeding, p. 79. Qingdao, China.

11. Damon M, Denieul K, Vincent A, et al. (2013) Associations between muscle gene expression pattern and technological and sensory meat traits highlight new biomarkers for pork quality assessment. Meat Sci 95, 744-754.

12. Larsson T, Morkore T, Kolstad K, et al. (2012) Gene expression profiling of soft and firm Atlantic salmon fillet. PLOS ONE 7, e39219.

13. Larsson T, Koppang EO, Espe M, et al. (2014) Fillet quality and health of Atlantic salmon (Salmo salar L.) fed a diet supplemented with glutamate. Aquaculture 426, 288-295.

14. Rochfort S (2005) Metabolomics reviewed: a new "omics" platform technology for systems biology and implications for natural products research. J Nat Prod 68, 1813-1820.
15. Picone G, Balling Engelsen S, Savorani F, et al. (2011) Metabolomics as a powerful tool for molecular quality assessment of the fish Sparus aurata. Nutrients 3, 212-227.

16. Sun J, Ando Y, Ahlbory-Dieker D, et al. (2013) Systems biology investigation to discover metabolic biomarkers of acetaminophen-induced hepatic injury using integrated transcriptomics and metabolomics. J Mol Biomark Diag S 1, 2.

17. Shi S, Shen Y, Zhao Z, et al. (2014) Integrative analysis of transcriptomic and metabolomic profiling of ascites syndrome in broiler chickens induced by low temperature. Mol Biosyst 10, 2984-2993.

18. Zhou M, Wang S, Zhao A, et al. (2012) Transcriptomic and metabonomic profiling reveal synergistic effects of quercetin and resveratrol supplementation in high fat diet fed mice. J Proteome Res 11, 4961-4971.

19. Sun Y, Yu K, Zhou L, et al. (2016) Metabolomic and transcriptomic responses induced in the livers of pigs by the longterm intake of resistant starch. J Anim Sci 94, 1083-1094.

20. Li H, Xie Z, Lin J, et al. (2008) Transcriptomic and metabonomic profiling of obesity-prone and obesity-resistant rats under high fat diet. J Proteome Res 7, 4775-4783.

21. Suravajhala P, Kogelman LJA \& Kadarmideen HN (2016) Multi-omic data integration and analysis using systems genomics approaches: methods and applications in animal production, health and welfare. Genet Sel Evol 48, 38.

22. Wei Z, Ma J, Pan X, et al. (2016) Dietary hydroxyproline improves the growth and muscle quality of large yellow croaker Larimichthys crocea. Aquaculture 464, 497-504.

23. Mevik BH \& Wehrens R (2007) The PLS package: principal component and partial least squares regression in R. J Stat Softw 18, 1-24.

24. Wickham H (2016) ggplot2: Elegant Graphics for Data Analysis. Houston, TX: Springer.

25. Zhang Q, Li B, Tang X, et al. (2012) An approach for metabonomics data analysis applied on the plasma of ginger water extract administered reserpine induced spleen deficiency rats. 2012 IEEE Symposium on Robotics and Applications (ISRA), pp. 511-513. Kuala Lumpur, Malaysia.

26. Mortazavi A, Williams BA, McCue K, et al. (2008) Mapping and quantifying mammalian transcriptomes by RNA-Seq. Nat Methods 5, 621-628.

27. Wang L, Feng Z, Wang X, et al. (2010) DEGseq: an R package for identifying differentially expressed genes from RNAseq data. Bioinformatics 26, 136-138.

28. Periago MJ, Ayala MD, López-Albors O, et al. (2005) Muscle cellularity and flesh quality of wild and farmed sea bass, Dicentrarchus labrax L. Aquaculture 249, 175-188.

29. Brüggemann DA \& Lawson MA (2005) The extracellular matrix of Gadus morhua muscle contains types III, V, VI and IV collagens in addition to type I. J Fish Biol 66, 810-821.

30. Sato K, Ando M, Kubota S, et al. (1997) Involvement of type $\mathrm{V}$ collagen in softening of fish muscle during short-term chilled storage. J Agric Food Chem 45, 343-348.

31. Kivirikko KI, Helaakoski T, Tasanen K, et al. (1990) Molecular biology of prolyl 4-hydroxylase. Ann N Y Acad Sci 580, 132-142.

32. Smith-Mungo LI \& Kagan HM (1998) Lysyl oxidase: properties, regulation and multiple functions in biology. Matrix Biol 16, 387-398.

33. Murphy G (2011) Tissue inhibitors of metalloproteinases. Genome Biol 12, 233.

34. Xu C, Wang C, Cai QF, et al. (2015) Matrix metalloproteinase 2 (MMP-2) plays a critical role in the softening of common carp muscle during chilled storage by degradation of type I and $\mathrm{V}$ collagens. J Agric Food Chem 63, 10948-10956. 
35. Aoki M, Suto K, Komatsu M, et al. (2012) Increasing effect of an oral intake of L-hydroxyproline on the soluble collagen content of skin and collagen fragments in rat serum. Biosci Biotechnol Biochem 76, 1242-1244.

36. Kwasek K, Terova G, Lee BJ, et al. (2014) Dietary methionine supplementation alters the expression of genes involved in methionine metabolism in salmonids. Aquaculture 433, 223-228.

37. Garrow TA (1996) Purification, kinetic properties, and cDNA cloning of mammalian betaine-homocysteine methyltransferase. J Biol Chem 271, 22831-22838.

38. Fukada SI, Shimada Y, Morita T, et al. (2006) Suppression of methionine-induced hyperhomocysteinemia by glycine and serine in rats. Biosci Biotechnol Biochem 70, 2403-2409.

39. Jong CJ, Azuma J \& Schaffer S (2012) Mechanism underlying the antioxidant activity of taurine: prevention of mitochondrial oxidant production. Amino Acids 42, 2223-2232.

40. Hansen SH, Birkedal H, Wibrand F, et al. (2015) Taurine and regulation of mitochondrial metabolism. Adv Exp Med Biol 803, 397-405.

41. Carneiro EM, Latorraca MQ, Araujo E, et al. (2009) Taurine supplementation modulates glucose homeostasis and islet function. J Nutr Biochem 20, 503-511.

42. Moon TW (2001) Glucose intolerance in teleost fish: fact or fiction? Comp Biochem Physiol B Biochem Mol Biol 129, 243-249.

43. Wion KL, Kirchgessner TG, Lusis AJ, et al. (1987) Human lipoprotein lipase complementary DNA sequence. Science 235, 1638-1641.

44. Schoonjans K, Peinado-Onsurbe J, Lefebvre AM, et al. (1996) PPARalpha and PPARgamma activators direct a distinct tissuespecific transcriptional response via a PPRE in the lipoprotein lipase gene. $E M B O J \mathbf{1 5}, 5336$.

45. Haunerland NH \& Spener F (2004) Fatty acid-binding proteins-insights from genetic manipulations. Prog Lipid Res $\mathbf{4 3}$, 328-349.
46. Martin G, Schoonjans K, Lefebvre AM, et al. (1997) Coordinate regulation of the expression of the fatty acid transport protein and acyl-CoA synthetase genes by PPAR $\alpha$ and PPAR $\gamma$ activators. J Biol Chem 272, 28210-28217.

47. Dobrzyn A \& Ntambi JM (2005) The role of stearoyl-CoA desaturase in the control of metabolism. Prostaglandins Leukot Essent Fatty Acids 73, 35-41.

48. Hong CH, Lee JM \& Kim KS (2004) Changes of nucleotides in the raw fishes during the aquarium storage. Korean J Food Sci Technol 36, 379-384.

49. Hong H, Regenstein JM \& Luo Y (2015) The importance of ATP-related compounds for the freshness and flavor of postmortem fish and shellfish muscle: a review. Crit Rev Food Sci Nutr 57, 1787-1798.

50. Alasalvar C, Taylor K, Öksüz A, et al. (2002) Comparison of freshness quality of cultured and wild sea bass (Dicentrarchus labrax). J Food Sci 67, 3220-3226.

51. Lakshmanan P, Antony P \& Gopakumar K (1996) Nucleotide degradation and quality changes in mullet (Liza corsula) and pearlspot (Etroplus suratensis) in ice and at ambient temperatures. Food Control 7, 277-283.

52. Gill TA (1990) Objective analysis of seafood quality. Food Rev Int $\mathbf{6}, 681-714$.

53. Heising J, Van Boekel M \& Dekker M (2014) Mathematical models for the trimethylamine (TMA) formation on packed cod fish fillets at different temperatures. Food Res Int 56, 272-278.

54. Zhao C, Pan Y, Ma L, et al. (2002) Assay of fish freshness using trimethylamine vapor probe based on a sensitive membrane on piezoelectric quartz crystal. Sens Actuators $B$ 81, 218-222.

55. Hamada-Sato N, Usui K, Kobayashi T, et al. (2005) Quality assurance of raw fish based on HACCP concept. Food Control 16, 301-307. 